\title{
Der Lockdown im Kanton Aargau
}

\section{Saskia Gauthiera, Daniel Eisenhart ${ }^{b}$, Patrick Laberke $^{c}$}

Institut für Rechtsmedizin Aargau, Kantonsspital Aarau

${ }^{a}$ Dr. med., Oberärztin; ${ }^{b}$ Dr. med., Chefarzt; ${ }^{c}$ Dr. med., Co-Abteilungsleiter Forensische Medizin und Oberarzt mbF

Es wurde befürchtet, dass während der Pandemie Fälle von häuslicher Gewalt und Suizide zunehmen könnten [1]. In der Forensik-Abteilung des Instituts für Rechtsmedizin Aargau (IRMAG) entstand tatsächlich der Eindruck, dass mehr Fälle häuslicher Gewalt und Suizide zu untersuchen waren als im gleichen Zeitraum in den vorherigen Jahren. Diese Einschätzung wurde in einer Studie untersucht, deren Ergebnisse im Folgenden vorgestellt werden.

In der Schweiz stand 2020 das öffentliche Leben im Rahmen der Corona-Pandemie zwischen dem 16. März und dem 11. Mai still. Schulen, Restaurants und Geschäfte waren auf behördliche Anweisung hin geschlossen, Versammlungen von über 5 Personen waren verboten, und die Einwohner wurden aufgefordert, zu Hause zu bleiben. Als Folge davon litt die Wirtschaft, es kam zu Hamsterkäufen von Toilettenpapier und Backhefe, zu einem Einbruch des Flugverkehrs und einem Ansturm auf Schweizer Naherholungsgebiete. Die im Folgenden vorgestellte retrospektive Studie gibt Aufschluss, ob die Fallzahlen von häuslicher Gewalt und/oder Suiziden während des Lockdowns im Kanton Aargau im Vergleich zu denselben Zeitperioden in den Vorjahren grosse Unterschiede aufweisen.

\section{Methode: Häufigkeitsanalyse}

Die elektronische Falldatenbank des Instituts für Rechtsmedizin Aargau wurde für den Zeitraum vom 16. März bis zum 11. Mai für die Jahre 2017-2020 nach forensisch-klinischen Untersuchungen und Suiziden durchsucht und eine Häufigkeitsanalyse durchgeführt. Das Signifikanzniveau wurde jeweils mit dem Chi-Quadrat-Test überprüft. An soziodemographischen Parametern wurden Alter und Geschlecht erhoben.

Tabelle 1: Anzahl der forensisch-klinischen Untersuchungen (KU) und der Suizide im Untersuchungszeitraum 16.3.-11.5. nach Jahr ( $p=$ Signifikanzniveau $<0,05)$.

\begin{tabular}{lll}
\hline Jahr & KU & Suizide \\
\hline $\mathbf{2 0 1 7}$ & 8 & 14 \\
\hline $\mathbf{2 0 1 8}$ & 23 & 24 \\
\hline $\mathbf{2 0 1 9}$ & 22 & 22 \\
\hline $\mathbf{2 0 2 0}$ & 36 & 22 \\
\hline $\mathbf{p}$ & .00076 & .70 \\
\hline
\end{tabular}

Bei den forensisch-klinischen Untersuchungen wurde sowohl nach Art der Untersuchung unterschieden (häusliche Gewalt, Körperverletzung, Untersuchung nach Sexualdelikt) als auch nach der Rolle der beteiligten Personen (geschädigt, tatverdächtig, beteiligt).

Bei den Legalinspektionen wurden nur Fälle eingeschlossen, bei denen kein Zweifel an einem Suizid bestand. Erhoben wurde die Art des Suizids, wie z.B. Erhängen oder Erschiessen, und, ob im Anschluss an die Legalinspektion eine Obduktion erfolgt war. Alle erhobenen Daten wurden vollständig anonymisiert.

\section{Ergebnisse}

In den Jahren 2017 bis 2020 wurden im IRMAG 784 forensisch-klinische Untersuchungen und 2279 amtsärztliche Leichenschauen (sog. Legalinspektionen) durchgeführt.

Tabelle 1 zeigt die Anzahl der jährlich zwischen dem 16. März und dem 11. Mai erfassten Fälle. Es fällt auf, dass 2020 deutlich mehr klinische Untersuchungen erfolgt waren als in den Jahren zuvor. Die Anzahl der untersuchten Suizide weist 2020 hingegen keinen deutlichen Anstieg im Vergleich zu den Vorjahren auf. In Tabelle 2 wird deutlich, dass die Fälle häuslicher Gewalt im definierten Untersuchungszeitraum starken Schwankungen unterlagen. Die Körperverletzungsdelikte sind 2020 im Vergleich zu den Vorjahren deutlich angestiegen. Bezogen auf den Mittelwert der Jahre 2017-2019 wurden im Jahr 2020 im Untersuchungszeitraum signifikant mehr forensisch-klinische Untersuchungen wegen Körperverletzungen durchgeführt. Die Anzahl der Legalinspektionen aufgrund von Suiziden unterschied sich während des Lockdowns nicht wesentlich von den Vorjahren. Tabelle 3 veranschaulicht jedoch, dass die assistierten Suizide im Untersuchungszeitraum im Vergleich zu den Vorjahren stark 
Tabelle 2: Anzahl der forensisch-klinischen Untersuchungen, gegliedert nach Schädigungsart im Untersuchungszeitraum.

\begin{tabular}{lllll}
\hline & HG & KV & SD & Anderes \\
\hline $\mathbf{2 0 1 7}$ & 4 & 4 & 0 & 2 \\
\hline $\mathbf{2 0 1 8}$ & 12 & 4 & 5 & 2 \\
\hline $\mathbf{2 0 1 9}$ & 2 & 14 & 4 & 4 \\
\hline $\mathbf{2 0 2 0}$ & 12 & 20 & 1 & .317 \\
\hline $\mathbf{p}$ & .057 & .00071 & .273 & \\
\hline
\end{tabular}

HG = häusliche Gewalt, $K V=$ Körperverletzungen, $S D=$ Sexualdelikt, $p=$ Signifikanzniveau $<0,05$.

abgefallen waren, während die absolute Anzahl der anderen Suizide leicht zugenommen hat. Dieser Unterschied ist jedoch nicht als signifikant zu erachten.

\section{Diskussion der Ergebnisse}

Der Kanton Aargau umfasst rund 685000 Einwohner und ist damit in der deutschsprachigen Schweiz der drittbevölkerungsreichste Kanton nach Bern und Zü-

\section{Gemäss der World Health Organization (WHO) kann die Pandemie das Risiko für häusliche Gewalt erhöhen.}

rich [2]. Das Institut für Rechtsmedizin Aargau führt seit 2017 im ganzen Kanton bei polizeilich zur Anzeige gebrachten Körperverletzungsdelikten forensischklinische Untersuchungen durch, wie z.B. bei häuslicher Gewalt, Sexualdelikten oder anderen Fällen interpersoneller Gewalt.

Ebenso werden bei allen aussergewöhnlichen Todesfällen im ganzen Kanton Legalinspektionen am Fundort durchgeführt. Als aussergewöhnliche Todesfälle gelten alle nicht-natürlichen Todesfälle, wie Suizide, Tötungsdelikte und Unfälle, sowie alle unklaren Todesfälle, bei denen die Todesart durch den leichenschauenden Arzt zunächst nicht eindeutig bestimmt werden kann und damit eine nicht-natürliche Todesart möglich ist.

Aufgrund dieser Umstände verfügt das IRMAG über umfassende Kenntnisse hinsichtlich der Fälle interpersoneller Gewalt und der aussergewöhnlichen Todesfälle im Kanton Aargau.

\section{Forensisch-klinische Untersuchungen}

Gemäss der World Health Organization (WHO) kann die aussergewöhnliche Situation der Pandemie das Risiko für häusliche Gewalt erhöhen. Stress, soziale Isolation, Verarmungsängste und der verminderte Zugang zu Sozial- und Gesundheitseinrichtungen sind nur ein
Teil der genannten Gründe [3, 4]. Mehrere Studien weltweit zeigen, dass es bereits zu einer Zunahme von häuslicher Gewalt im Rahmen der behördlichen Covid19-Massnahmen gekommen ist [4-6].

Während des Lockdowns in der Schweiz im Frühjahr 2020 wurden signifikant mehr forensisch-klinische Untersuchungen im Kanton Aargau durchgeführt als im Vergleich zu den Vorjahren, wobei insbesondere Untersuchungen wegen Körperverletzungen, im Sinne von Prügeleien und Messerstechereien, angestiegen waren. Bezüglich der Fälle von häuslicher Gewalt lässt sich zwar ein leichter Anstieg feststellen, dieser verfehlt jedoch knapp das Signifikanzniveau von $p<0,05$. Einschränkend ist hierzu allerdings die vermutlich hohe Dunkelziffer häuslicher Gewalt anzuführen, wobei es im Lockdown aufgrund der sozialen Isolation und behördlichen Anordnung, zu Hause zu bleiben, dem Opfer möglicherweise noch schwerer gemacht wurde, sich der Kontrolle des Aggressors zu entziehen und eine Anzeige bei der Polizei zu erstatten.

\section{Suizide}

In Hinblick auf das Suizidrisiko wird angenommen, dass Pandemien durch soziale und ökonomische Aspekte zu einem erhöhten Suizidrisiko führen können, wie bereits in den USA während der Grippe-Epidemie 1918/19 und in Hongkong während der SARS-Epidemie $2003[7,8]$ beobachtet werden konnte. Soziale Isolation, Vereinsamung, Angst, Verlust der Arbeit und finanzielle Probleme sind nur einige der vielen Gründe, die im Rahmen der Corona-Pandemie zu einem erhöhten Suizidrisiko führen können.

Es wird angenommen, dass Pandemien durch soziale und ökonomische Aspekte zu einem erhöhten Suizidrisiko führen können.

In unserem Untersuchungsgut ist kein signifikanter Unterschied in den absoluten Fallzahlen der Suizide $\mathrm{zu}$ erkennen. Es fand aber 2020 eine leichte Verschiebung hin zu den nicht-assistierten Suiziden statt, wobei dieser Unterschied jedoch nicht signifikant ist. Ein Grund hierfür könnte sein, dass aufgrund der behördlichen Massnahmen auf nicht absolut dringliche Freitodbegleitungen verzichtet wurde. Da der Untersuchungszeitraum dieser Studie zu Beginn der Pandemie lag, könnten sich allfällige Auswirkungen wirtschaftlicher Schäden auf die Suizidrate und/oder die interpersonelle Gewalt auch erst zu einem späteren Zeitraum manifestieren. Weitere Studien über einen längeren Zeitraum mit detaillierter Erfassung der Suizidgründe könnten hier Aufschlüsse liefern. 
Tabelle 3: Anzahl der Suizide, gegliedert nach Methode im Untersuchungszeitraum.

\begin{tabular}{llllllll}
\hline & Erhängen & Ertrinken & $\begin{array}{l}\text { Er- } \\
\text { schiessen }\end{array}$ & $\begin{array}{l}\text { Zug } \\
\text { Höhe }\end{array}$ & $\begin{array}{l}\text { Sturz aus } \\
\text { AS }\end{array}$ & Anderes \\
\hline $\mathbf{2 0 1 7}$ & 5 & 1 & 1 & 1 & 0 & 4 & 2 \\
\hline $\mathbf{2 0 1 8}$ & 1 & 1 & 2 & 2 & 3 & 14 & 0 \\
\hline $\mathbf{2 0 1 9}$ & 4 & 2 & 2 & 1 & 2 & 11 & 0 \\
\hline $\mathbf{2 0 2 0}$ & 3 & 3 & 4 & 4 & 2 & 6 & 0 \\
\hline $\mathbf{p}$ & .872 & .275 & .177 & .102 & .827 & .283 & \\
\hline
\end{tabular}

AS $=$ assistierter Suizid, $p=$ Signifikanzniveau $<0,05$.

\section{Einschränkungen der Studie}

Die vorliegende Studie umfasste lediglich rechtsmedizinisch untersuchte Fälle im Zeitraum vom 16. März bis zum 11. Mai der Jahre 2017-2020. Die nicht polizeilich gemeldeten Fälle von körperlicher Gewalt wurden somit rechtsmedizinisch nicht erfasst, und es handelt sich um einen kurzen Zeitraum, der grossen Schwankungen unterliegen kann.

Folgestudien über einen längeren Zeitraum sowie in Zusammenarbeit mit den kantonalen Opferhilfestellen und der Polizei könnten insbesondere in Hinblick auf interpersonelle Gewalt, die nicht zur Anzeige gebracht wurde, einen detaillierten Einblick über die Auswirkungen der Corona-Pandemie im Kanton Aargau geben.

\section{Prävention ist wichtig}

Unsere Studienergebnisse zeigen, dass es im Kanton Aargau durch den Lockdown und die damit einher-

\section{Das Wichtigste in Kürze}

- Im Kanton Aargau ist es 2020 während des Lockdowns zu einer Zunahme von Körperverletzungsdelikten gekommen. Die befürchtete Zunahme häuslicher Gewalt konnte durch die Fallzahlen nicht objektiviert werden.

- Mit Ausnahme des assistierten Suizids nahm im Aargau 2020 während des Lockdowns auch die Zahl der Suizide leicht zu, mutmasslich im Zusammenhang mit den behördlichen angeordneten Massnahmen. Künftig sollte ein besonderes Augenmerk auf die Prävention gelegt werden. gehenden Einschränkungen tatsächlich zu einer Zunahme von Körperverletzungsdelikten gekommen ist. Der subjektive Eindruck einer Zunahme häuslicher Gewalt konnte aufgrund der Fallzahlen jedoch nicht objektiviert werden.

In der Zusammenschau mit der Literatur konnte auch im Kanton Aargau eine Zunahme interpersoneller Gewalt und von Suiziden, mutmasslich in Zusammenhang mit dem behördlich angeordneten Lockdown, festgestellt werden, so dass zukünftig ein besonderes Augenmerk auf präventive Massnahmen gelegt werden sollte.

\section{Literatur}

1 www.aargauerzeitung.ch/schweiz/ich-gehe-leider-davon-ausdass-sich-die-situation-nicht-rasch-bessert-coronakrise-fuehrtzu-mehr-haeuslicher-gewalt-139811000. Accessed 7.12.2020.

2 Kanton Aargau in Zahlen. www.ag.ch/de/weiteres/portrait/ zahlen und fakten/zahlen und fakten.jsp. Accessed 7.12.2020.

3 WHO. Covid-19 and violence against women. www.who.int/ reproductivehealth/publications/emergencies/COVID-19-VAWfull-text.pdf. Accessed 20.10.2020.

4 UN Women, 2020. Violence Against Women and Girls: the Shadow Pandemic. Retrieved May 3, 2020, from United Nations: www. unwomen.org/en/news/stories/2020/4/statement-ed-phumzileviolence-against-women-during-pandemic. Accessed 7.12.2020.

5 Boserup B, McKenney M, Elkbuli A. Alarming trends in US domestic violence during the COVID-19 pandemic. American Journal of Emergency Medicine, https://doi.org/10.1016/j

6 Fraser E. Impact of COVID-19 pandemic on violence against women and girls. Development UAftDoI; 2020

7 Gunnell D, Appleby L, Arensman E, Hawton K, John A, Kapur N, Khan M, O'Connor RC, Pirkis J. COVID-19 Suicide Prevention Research Collaboration. Suicide risk and prevention during the COVID-19 pandemic. Lancet Psychiatry. 2020 Jun;7(6):468-71.

8 Sher L. The impact of the COVID-19 pandemic on suicide rates. QJM. 2020 Oct 1;113(10):707-12.

\section{L'essentiel en bref}

- Dans le canton d'Argovie, une augmentation des lésions corporelles a été constatée pendant le semi-confinement en 2020. La hausse redoutée de la violence domestique ne s'est pas concrétisée avec le nombre de cas.

- Le nombre de suicides - sauf le suicide assisté a également légèrement augmenté en Argovie en 2020 pendant le semi-confinement, vraisemblablement en lien avec les différentes mesures ordonnées. Une attention particulière devra à l'avenir être accordée à la prévention. 